\title{
Concurrent chemoradiotherapy for locally advanced squamous cell carcinoma of the cervix in a uterus didelphys with vaginal septum
}

\author{
Yuko Kaneyasu, MD, PhD ${ }^{12}$, lemasa Koh, MD, PhD ${ }^{3}$, Hisaya Fujiwara, MD, PhD ${ }^{4}$, Eiji Hirata, MD, PhD ${ }^{3}$ \\ Yoshiki Kudo, MD, PhD³, Koji Arihiro, MD, PhD5, Norio Miharu, MD, PhD6, Tomio Nakagawa, MD, PhD', \\ Ippei Takahashi, MD, PhD², Yuki Takeuchi, MD², Nobuki Imano, MD², Ikuno Nishibuchi, MD, PhD², Takeo Nakashima, PhD7, \\ Daisuke Kawahara, PhD 7 , Yoshimi Ohno, MS77, Yasushi Nagata, MD, PhD² \\ 'Department of Radiation Oncology, National Hospital Organization Fukuyama Medical Center, Hiroshima, Japan, ${ }^{2}$ Department of Radiation \\ Oncology, Graduate School of Biomedical Sciences, Hiroshima University, Hiroshima, Japan, ${ }^{3}$ Department of Obstetrics and Gynecology. \\ Hiroshima University, Hiroshima, Japan, ${ }^{4}$ Department of Obstetrics and Gynecology, Japan Organization of Occupational Health and \\ Safety. Chugoku Rosai Hospital, Hiroshima, Japan, ${ }^{5}$ Department of Anatomical Pathology. Hiroshima University Hospital, Hiroshima, Japan, \\ ${ }^{6}$ Department of Obstetrics and Gynecology. Chuden Hospital, Hiroshima, Japan, ${ }^{2}$ Section of Radiation Therapy. Department of Clinical \\ Support, Hiroshima University Hospital, Hiroshima, Japan
}

\begin{abstract}
In November 2011, a 61-year-old woman was diagnosed with squamous cell carcinoma (SCC) of the cervix in a uterus didelphys with vaginal septum. The patient was diagnosed with Fédération Internationale de Gynécologie et d'Obstétrique (FIGO) stage IIB because of infiltration to the left parametrium without infiltration to the pelvic wall. The patient was treated with external-beam radiotherapy (EBRT) and brachytherapy (BT), using concomitant chemotherapy with cisplatin. A total of $50 \mathrm{~Gy}$ were delivered (2 Gy/fraction/day) to the pelvis, with a central shield after 40 Gy. The patient was treated four times with BT (6 Gy $\times 4$ fractions), with tandem and ovoid applicators inserted once to the left side; tandem to the left side and ovoid bilaterally were inserted twice; and tandem to the right side and ovoid bilaterally were inserted once. Six years and 8 months after the start of treatment, the patient had had no relapse or severe late adverse effects. For accurate diagnosis and optimal treatment of the uterus didelphys, careful interview and pelvic examination at initial diagnosis of a patient are very important.

J Contemp Brachytherapy 2019; 11, 2: 180-188 DOI: https://doi.org/10.5114/jcb.2019.84506
\end{abstract}

Key words: cervical cancer, brachytherapy, uterus didelphys, vaginal septum, concurrent chemoradiotherapy.

\section{Purpose}

Uterine and vaginal malformations due to abnormal development of the Müllerian ducts are classified as non-syndromic minor malformations. These include those found during systematic examination for infertility as well as genital organs that are completely blocked, with early symptoms during the menstrual period. Although it is difficult to determine the frequency of uterus didelphys accurately, which varies according to reports, the frequency has been reported to be about one in 10,000 (range, about 1 in 1,000 to 1 in 30,000) of obstetric admissions in general $[1,2]$. There is no standard treatment for cervical cancer occurring in uterine malformations. We report a rare case of cervical cancer that occurred in uterus didelphys (with double vagina and uterus), cured with concurrent chemoradiotherapy (CCRT).

\section{Case report}

The patient was a 61-year-old woman, gravida 0 , para 0 . She was referred to the Hiroshima University Hospital on October 19, 2011, with atypical genital bleeding in the last 4 months. She had been diagnosed in our hospital to have uterus didelphys with double vagina and uterus at the time of examination for infertility at the age of 29 . Her past history revealed previous surgery for hip osteoarthritis at age of 20. Her family history was unremarkable. The patient was found to have normal vulva. No renal or any other abnormalities were present. Several pelvic examinations in our hospital showed cervical tumor in the uterus didelphys, with a complete vaginal septum extending to the introitus. However, the uterus didelphys had not been observed by the referring hospital, but initially at our hospital. Because the patient complained of pain during
Address for correspondence: Yuko Kaneyasu, MD, PhD, Department of Radiation Oncology, National Hospital Organization Fukuyama Medical Center, 4-14-17 Okinogami-cho, Fukuyama, Hiroshima, 720-8520 Japan, phone: +81-84-922-0001, fax: +81-84-931-3969, - e-mail: kaneyasuyuko@gmail.com
Received: 04.08 .2018

Accepted: 03.03.2019

Published: 29.04.2019 
pelvic examination, biopsy was performed under intravenous anesthesia, and the initial pathological result of a cervical tumor in one cervix was CIN 3. Furthermore, pelvic examination, hysteroscopy, and biopsy were performed under intravenous anesthesia in the operating room. At that time, the gynecologic oncologist observed the uterus didelphys with vaginal septum for the first time. There were easy bleeding tumors on each cervix at Cusco's examination. Macroscopic findings, hysteroscopic findings, and the vaginal septum at BT are shown in Figure 1. The tumor at the left cervix was circumferential, whereas the tumor at the right cervix had a left side deviation (septum side) at the hysteroscopy. Histopathological findings of the bilateral cervical biopsy specimens indicated non-keratinizing squamous cell carcinoma. Human papillomavirus (HPV) infection was not investigated.

Initially, in our department, sounding of the right uterus was impossible, while the sounding length of the left uterus was only $2 \mathrm{~cm}$. The tumor on the left side was larger than that on the right side, and pelvic examination revealed that the left side tumor had invaded the left parametrium. No invasion was observed in either of the vaginal walls.

Positron emission tomography-computed tomography (PET-CT) of the head to the pelvis, CT of the chest to the pelvis, and magnetic resonance imaging (MRI) of the pelvis were performed. These showed a tumor with a maximum diameter of $43 \mathrm{~mm}$ (more to the left side), spread across the bilateral uterine cervices, and with-
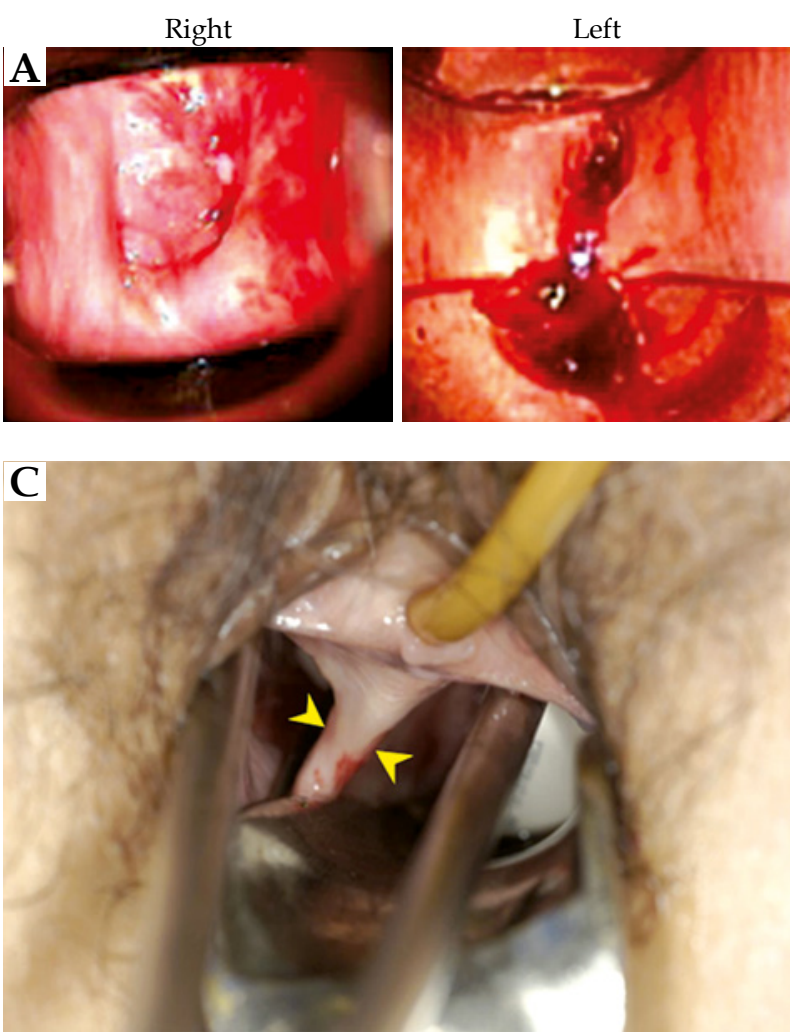

out pelvic and paraaortic lymph node enlargement. According to the Fédération Internationale de Gynécologie et d' Obstétrique (FIGO) classification [3], the tumor was stage IIB. The serum level of the tumor marker, squamous cell carcinoma (SCC) antigen was elevated to $7.7 \mathrm{ng} / \mathrm{ml}$ (normal range, $\leq 1.5 \mathrm{ng} / \mathrm{ml}$ ). The serum level of the tumor marker, carcinoembryonic antigen (CEA), and blood counts were within the normal ranges. The pre-treatment MRI images of the tumor in bilateral uteri are shown in Figure 2. The tumor on the right side of the uterus was localized in the cervix. On the other hand, the tumor on the left side showed extrauterine invasion (Figure 2). Figure 3 shows the MRI before and during treatment, from bilateral uterine bodies to fundus and vagina.

In addition to the carcinoma of the uterus and vaginal didelphys, based on the pelvic examination and imaging diagnosis, the tumor invasion range in the patient was considered to be wide (stage IIB, with left parametrial involvement, tumor size of $43 \mathrm{~mm}$ ). This finding was judged to be an indication for radiotherapy rather than surgery. The type of uterine didelphys in the patient according to Jarcho classification [4] was categorized as class 1. Uterus didelphys bicollis (septate vagina) according to the American Society of Reproductive Medicine classification [5] was class III. Radiotherapy (RT) was administered via a combination of external beam radiotherapy (EBRT) to the pelvic cavity and intracavitary irradiation. EBRT was given to the whole pelvis using four-field box technique with a 10-MV linear accelerator unit. The daily fraction

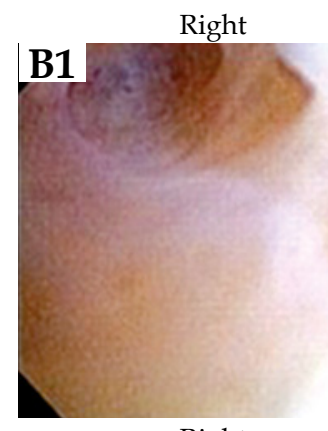

Right

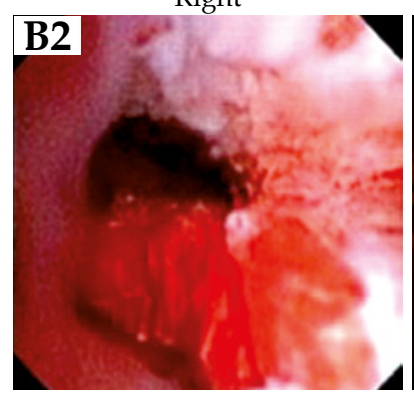

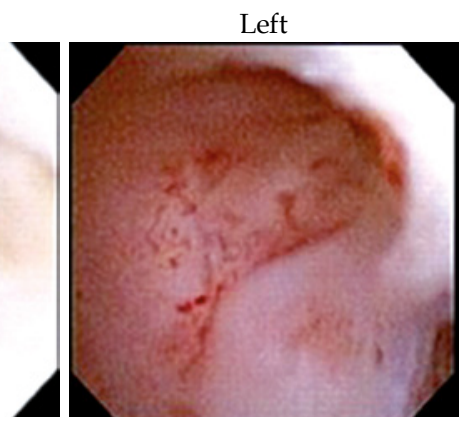

Left

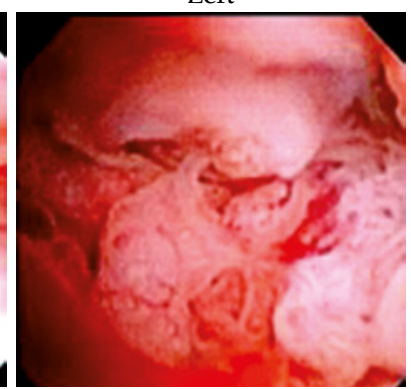

Fig. 1. A) Macroscopic findings of the right and left uterine cervices at Cusco's examination. B) Findings on hysteroscopy. B-1) Uterine body (right and left): Each tumor had not invaded the uterine body. B-2) Uterine cervix: The left tumor is larger than the right one. The tumor at the left cervix was circumferential, the tumor at the right cervix was on the left side of the deviation. C) Bilateral vagina with ovoid applicator insertion. These two arrowheads point to the vaginal septum extending to the introitus 

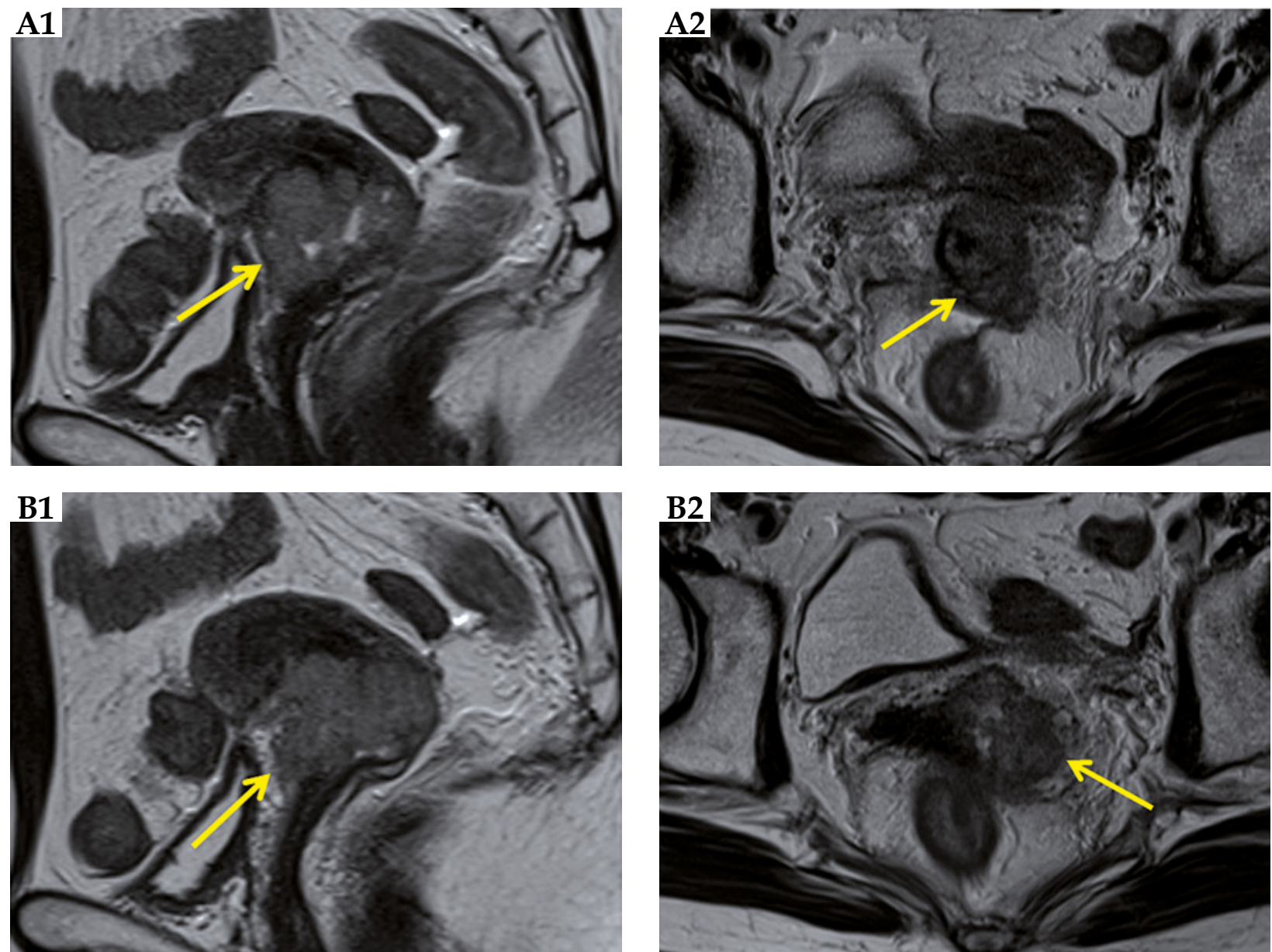

Fig. 2. Pelvic magnetic resonance imaging (MRI) before treatment. A-1) Right uterine tumor is confined to the cervix (arrow). A-2) Tumor of the right cervix. B-1) Left uterine tumor invades to the surrounding tissue beyond the cervix. B-2) Tumor of the left cervix

size was $2.0 \mathrm{~Gy}$, with 5 fractions weekly. Details of the radiation field were reported previously [6]. A central shield was used after $40 \mathrm{~Gy}$, with external whole pelvic irradiation. From November 22 to December 29, 2011, the total dose administered was $50 \mathrm{~Gy}$ ( 25 fractions/38 days). High-dose-rate intracavitary BT (HDR-ICBT) was given with ${ }^{192}$ Ir micro-Selectron (Nucletron, The Netherlands). The dose at point A was 6 Gy per fraction, 1 fraction per week, and the number of fractions was 4 , for a total dose of 24 Gy on December 20 and 27, 2011 and January 4 and 10, 2012. Total treatment time of RT was 50 days. With the cooperation of the gynecological oncologists, ultrasound guidance was used, and it was confirmed that the tip of the tandem reached the fundus of the uterus. Because each vagina was very narrow and the patient complained of pain at the time of application, all 4 applications were performed under intravenous anesthesia. Oxygen was administered at 3 liters per minute with an oxygen mask. At the time of application, vaginal packing with gauzes containing radio-opaque fiber were used to show the state and thickness of the rectal and bladder sides packing. Tandem and ovoid applicators were used for each application. The ovoid size was $30 \times 20 \times 14 \mathrm{~mm}$ in diameter. At the first treatment, only the left side of the uterus was treated with tandem and ovoid applicators.
Because the vagina was very narrow, we could not interpose the flange with the two ovoid applicators (these two applicators deviated to the foot side from the flange). The sounding length to the fundus of the left uterus was only $3.5 \mathrm{~cm}$. At the second to third treatments, a tandem was inserted to the left uterus, whereas two ovoid applicators were placed at the right and left vaginal fornices. By the second time, tandem insertions into the uterine cavities on both sides were attempted. The sounding length of the right uterus was $4 \mathrm{~cm}$. However, when a tandem was first inserted on the right side, the insertion on the left side became insufficient because of the narrowing between the bilateral cervical canals. Therefore, a tandem was inserted only on the left side containing the large tumor. We were worried about the insufficient dose to the whole uterus on the right. Therefore, at the last treatment, a tandem and a right-side ovoid were inserted to each of the right uterus and the right vagina, respectively, and a left ovoid was inserted to the left vagina. Dose distributions of each ICBT are shown Figure 4 [7]. Although it is an approximation, most of the tumor volumes before treatment with each ICBT (red dashed lines drawn by referring to the pre-treatment MRI image) are surrounded by 6 Gy isodose curves. In addition, the tumor volume just after the second ICBT (yellow dashed line drawn by referring 

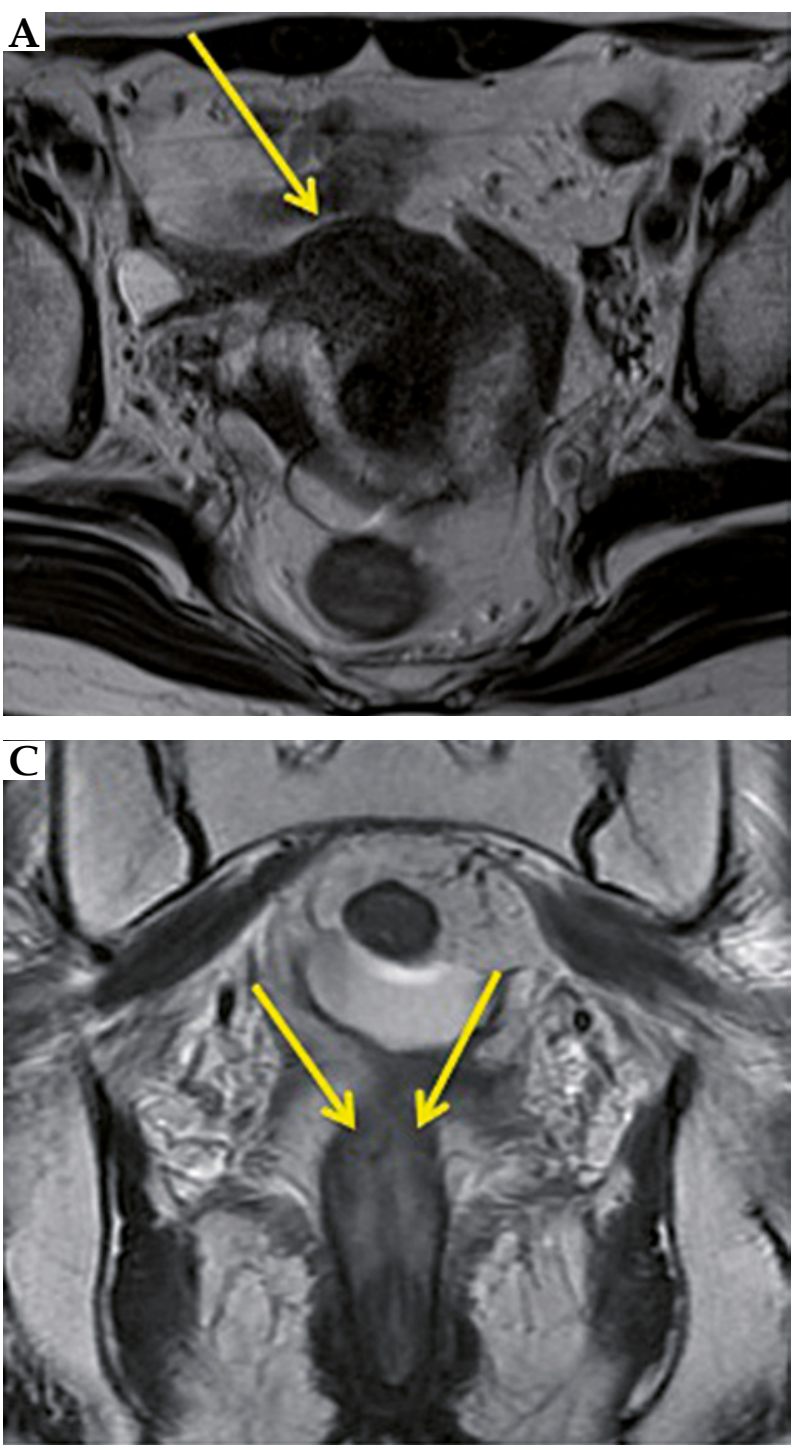

to MRI just after the second ICBT) was completely enclosed within the $6 \mathrm{~Gy}$ isodose curve.

The distances between the bilateral ovoid sources at each application were 1.2, 3.5, 3.6, and 3.2 (mean, 2.9) cm, respectively. We regarded the thicknesses of X-ray gauze packing as the distance between ovoid and vaginal posterior wall for each ICBT. Those measurements were $0,4.5$, 2.6, and 0 (mean, 1.8 ) $\mathrm{mm}$. Orthogonal X-rays were used for the calculation of these distances.

The rectal dose was evaluated using a rectal dosimeter, and the reference dose point as well as the bladder dose were defined according to the recommendations of the ICRU 38 report [8]. There were five measurement points based on the rectal dosimeter, and the maximum one was evaluated. The mean doses at point $\mathrm{A}$, at the rectum by the ICRU 38, at the rectum by calculation, at the rectum by dosimeter measurement, and at the bladder by the ICRU 38 per fraction were 6.1 Gy, 7.7 Gy, 5.2 Gy, 4.2 Gy, and 7.8 Gy, respectively. The total doses of these five points were 72.7, 107.9, 74.1, 64.0, and 107.6 $\mathrm{Gy}_{\mathrm{EOD} 2}$, respectively. The patient received six courses of concurrent chemotherapy,

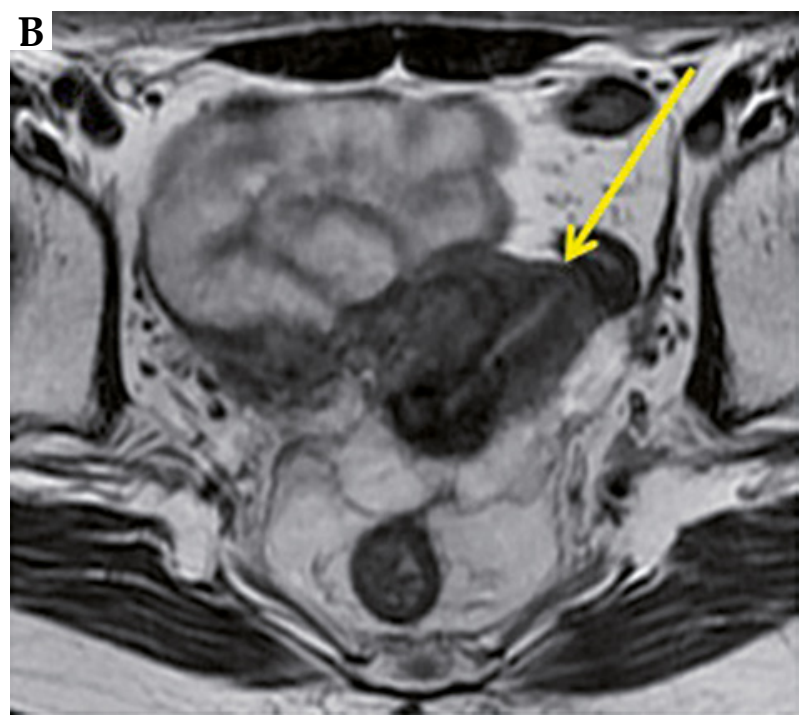

Fig. 3. MRI before and during treatment. A) Right uterine body to the fundus. B) Left uterine body to the fundus. C) Bilateral vaginas

with $30 \mathrm{mg} / \mathrm{m}^{2}$ of weekly cisplatin (CDDP). No acute adverse events such as diarrhea occurred in this patient.

The primary tumor response was the complete response (CR) by pelvic examination just after CCRT; and by pelvic images on MRI and CT at 4 months after CCRT (Figure 5). The serum SCC level returned to within normal limits, with a value of $0.8 \mathrm{ng} / \mathrm{ml}$.

Four years and three months after CCRT, the patient developed a late rectal complication, grade 1 radiation proctitis, which was graded according to the Radiation Therapy Oncology Group/European Organization for Research and Treatment of Cancer late radiation morbidity scoring criteria [9]. The patient has been followed-up without treatment because she has no anemia. The patient was alive and well with no evidence of recurrence or any other late complications 6 years and 8 months after the start of CCRT.

\section{Discussion}

It is very rare to encounter uterine malformations in daily gynecologic cancer examinations. The treatment of 


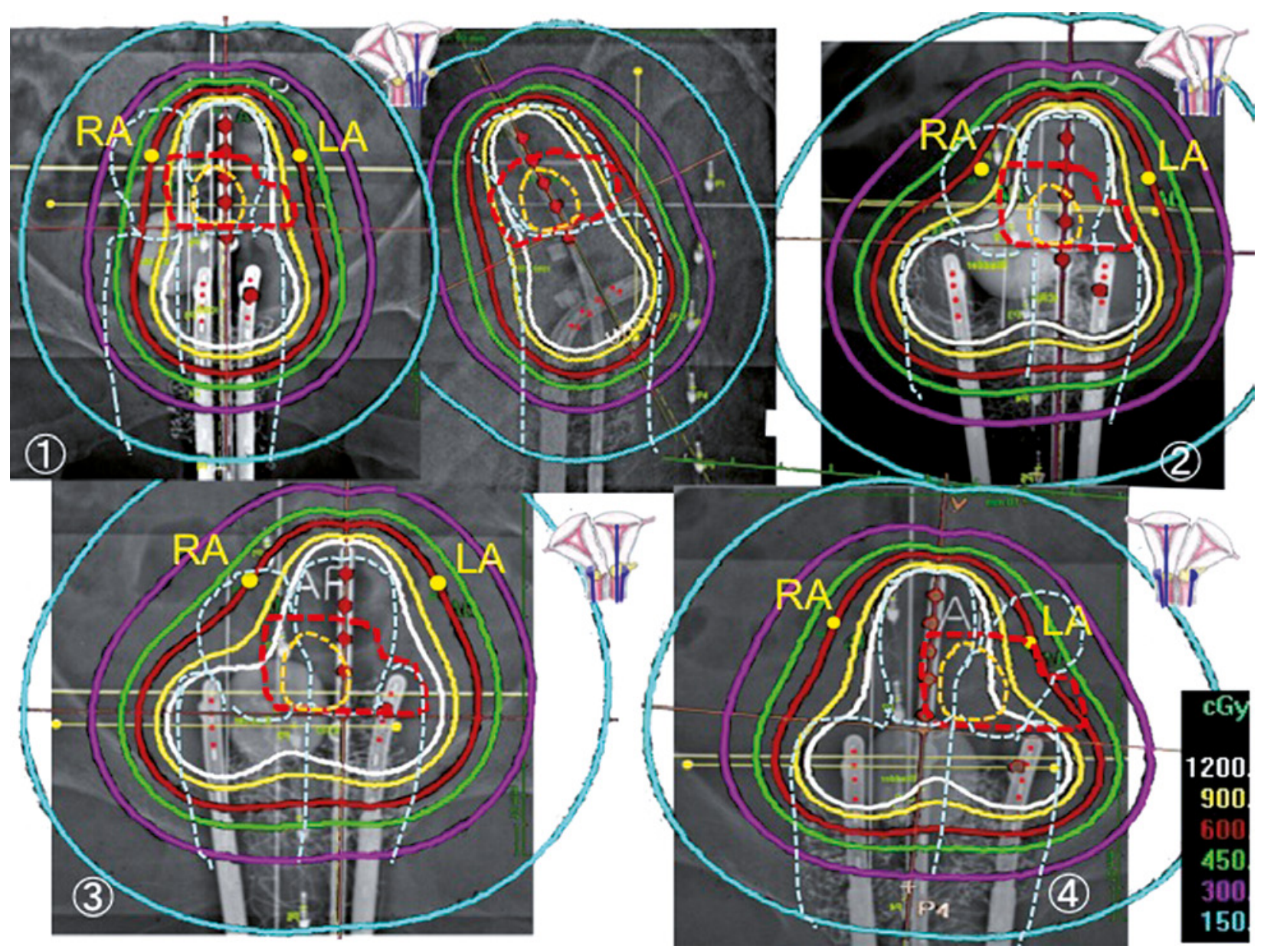

$R A$ - right point $A, L A$ - left point $A$

White dashed lines - the uterus didelphys contours with double vagina and uterus

Red dashed lines - the tumor contours drawn with reference to pre-treatment MRI image.

Yellow dashed lines - the tumor contours drawn with reference to MRI image just after the second ICBT.

Fig. 4. Dose distributions of each of the four brachytherapy.

The small schemas on the upper left side of the figures were drawn, modified using reference [7]
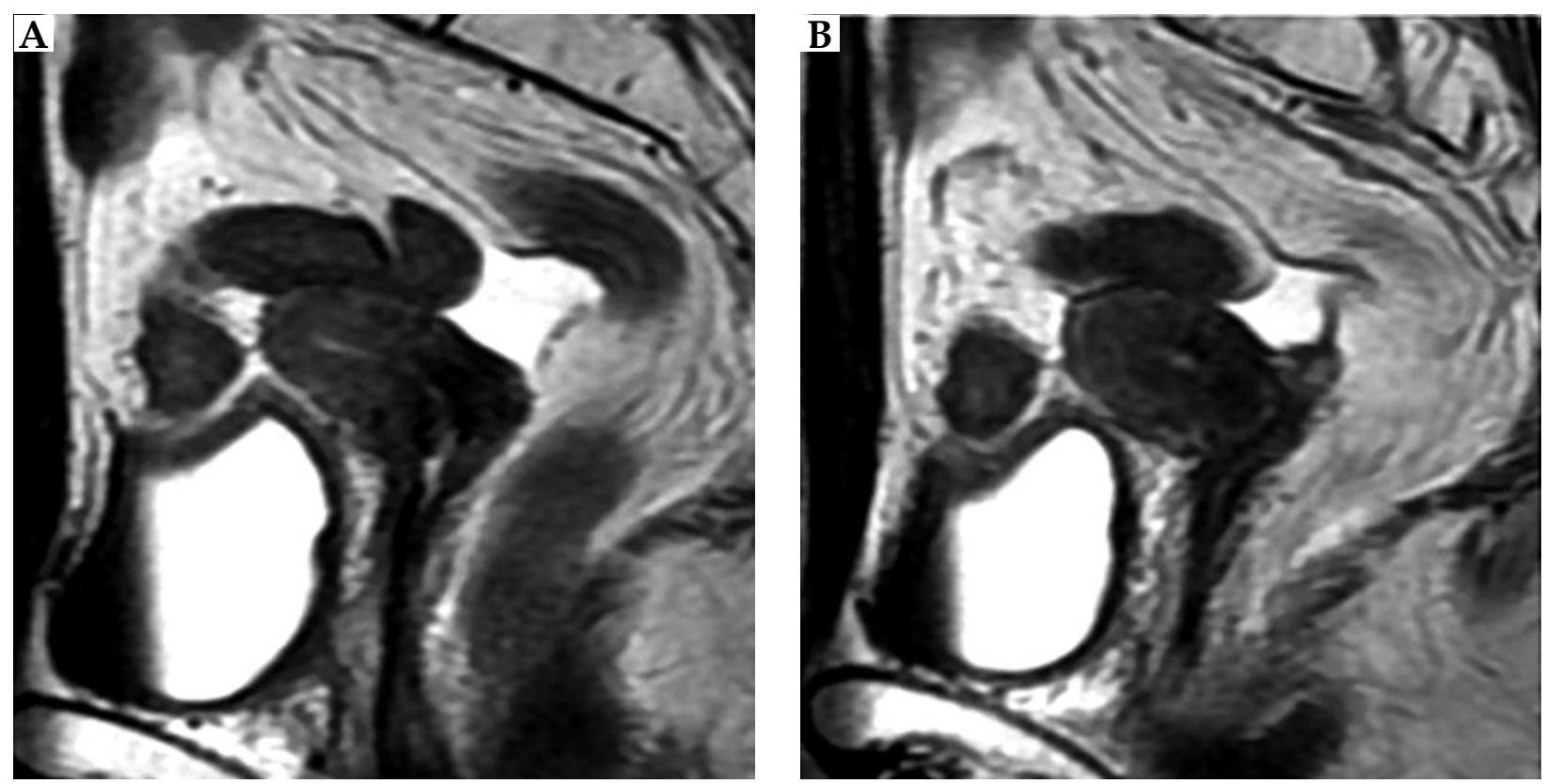

Fig. 5. MRI taken at 4 months after the treatment. A) Right uterus. B) Left uterus 
a patient with carcinoma of the cervix in a uterus didelphys is not described or established in textbooks, but it is only seen in case reports. The individual patient treatment is determined by the judgment of each physician. Therefore, we believe that it is meaningful for such physician to report the treatment method. Then, if physicians encounter similar cases, they can discuss the treatment method whether radiotherapy is possible and how to irradiate concretely. These actual experiences with cases are very valuable, and the physicians can refer to them in the literature. Patients can be treated similarly based on these experiences and planned for further treatment. If these cases are accumulated, the treatment method for a patient can be established. We can then compare radiotherapy (RT) with surgery by referring to the following surgical cases. Several reports of carcinoma in a uterus didelphys, which were treated surgically with or without preoperative RT were recognized until now, including a case where one side was non-cancerous, while the other side had cervical or endometrial cancer $[10,11,12,13,14,15,16,17,18]$. In total, there were only seven radiotherapy cases of cervical cancer in uterus didelphys reported until now (Table 1). It is very important to discuss the treatment method whether RT or surgery for the uterus didelphys at diagnosis.

The diagnosis of cervical cancer in uterus didelphys with vaginal septum is very difficult; however, very important. When the cervical cancer of the cervix is identified in only one side, leaving the other side unidentified, the dose for the other tumor and uterus may be insufficient, because only one uterus would receive ICBT. Thus, in a patient with such inadequate dose prescribed for the other cervical cancer, a risk of recurrence may occur. A sufficient inquiry during medical examination of a patient is particularly important for the diagnosis of cervical cancer in uterus didelphys. There are uterine malformations, which adopt an autosomal recessive or dominant genetic form. The uterus didelphys may complicate urinary system malformations, skeletal system, and auditory diseases among others. According to a report, in 5-10\% of infertile patients, uterus didelphys is implicated. With a history of infertility or nulliparity, with malformations mentioned above (in a patient or her family members), we should carefully conduct a pelvic examination of the patient, with the existence of a uterus didelphys in mind. This current case was also an infertile patient, with complications of the hip osteoarthritis. Moreover, in a case with a complete vaginal septum extending to the introitus, as in this patient, we may not notice the existence of another vagina and uterus without careful observation of the vulva and vagina before Cusco's examination. The practical clinical point in the management of a patient with abnormal bleeding that the finding of a vaginal septum impels careful search for a second cervix has been emphasized [17].

As with this patient, the uterus didelphys was diagnosed at the time of examination for infertility. If the patient had not undergone scrutiny for infertility, the uterus didelphys might not have been discovered. In that case, without careful internal examination by a physician, uterus didelphys would not have been diagnosed.

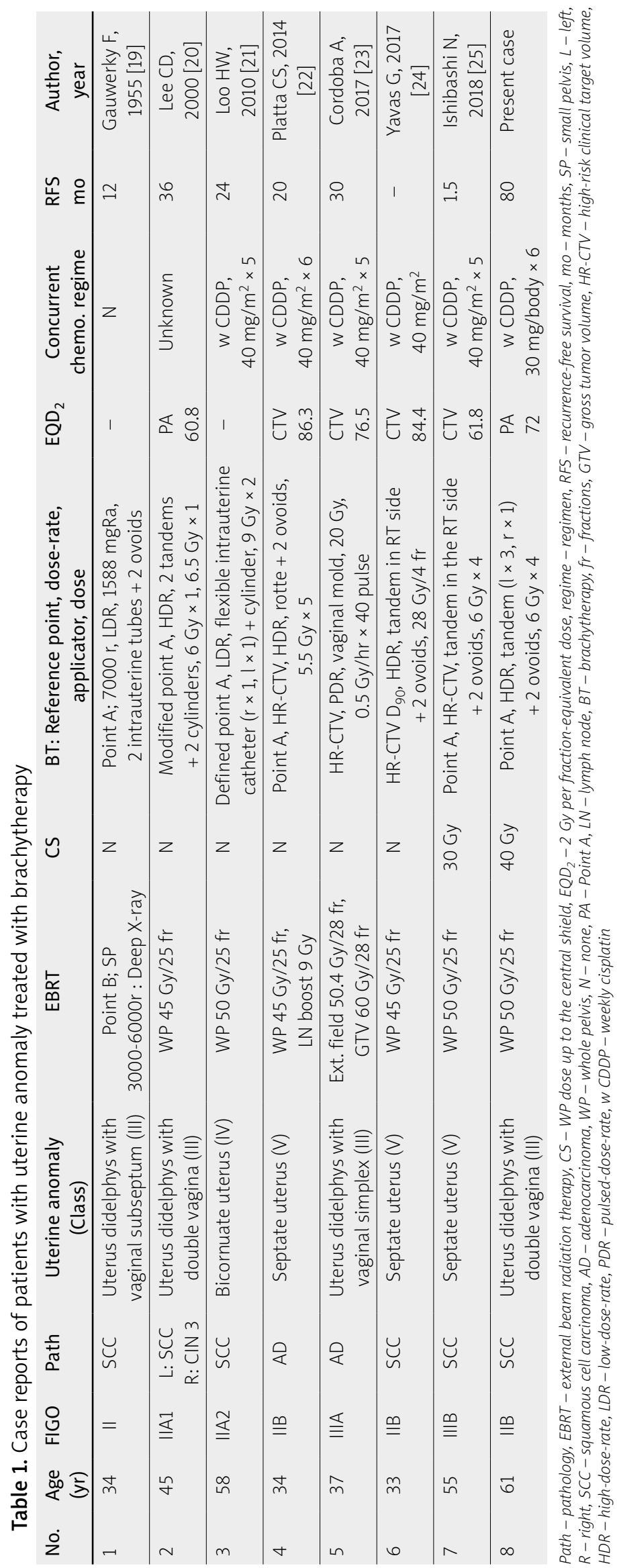


The role of HPV infection has become clear in cervical cancer occurrences. The role of transmissible coital factors in the causation of invasive carcinoma of the cervix have been reported $[11,12,14]$. In our case, two possibilities of carcinogenesis are conceivable. First, bilateral uterine cervix was exposed during sexual intercourse, with each individually developing uterine cervical carcinoma. Second, only one side of the uterine cervix was exposed during sexual intercourse, and the tumor that developed on one side invaded the other side. If we had examined for the HPV status of the bilateral uterus, and each type was different, there was the possibility that they might have been experienced carcinogenesis separately. However, since mixed infections do exist, the actual mechanism of the carcinogenesis of the bilateral uterus may be unknown.

Surgery was judged to be difficult, because the present patient had a stage IIB locally advanced cancer, which was larger than $40 \mathrm{~mm}$ in size, with left parametrium invasion. It was difficult to diagnose whether the right and left tumors were independent [10]. The left tumor was larger than the right tumor at pelvic examination, in hysteroscopic findings, and at MRI. Thus, there is the possibility that the tumor on the left grew in continuity and invaded the right side of uterine cervix.

As mentioned above, to the best of our knowledge, there were only seven previously reported cases of carcinoma of the cervix in patients with uterine anomalies treated with radical radiotherapy (Table 1). In six institutions in countries other than Japan, central shields were not used. For the first time, Gauwerky reported BT as a treatment for FIGO stage II SCC of the cervix with uterus didelphys with vaginal subseptum [19]. Two intrauterine tubes and 2 ovoids were used for double uterus and bilateral vaginal fornix. Lee et al. reported BT as a treatment for FIGO stage IIA1 SCC of the cervix with uterus didelphys, class III Müllerian duct anomaly [20]. Instead of using the traditional point $\mathrm{A}$, the prescription point was $2 \mathrm{~cm}$ superior to the mean position of the small metallic flange located at each cervical os at the midline between the two intra-uterine tubes. Loo et al. reported a stage IIA2 squamous cell carcinoma of a bicornuate bicollis uterus (class IV Müllerian duct anomaly) treated with bilateral intracavitary BT [21]. For fraction 1, the intrauterine catheter was inserted into the right uterine canal, and a radio-opaque marker was inserted into the left uterine canal. The right Manchester A point was calculated as tilted, with the angulated intrauterine source train as well as tilted with the angulated radio-opaque marker in the contralateral uterine canal. This process was repeated for the opposite canals for the second fraction. Three dimensional CT-based planning was performed in four institutions [22,23,24,25]. Platta et al. reported a stage IIB adenocarcinoma of a septate uterus (class V Müllerian duct anomaly), treated with Rotte- $Y$ applicators and 2 ovoid applicators [22]. Five fractions of 5.5 Gy each were delivered to Point A with CT-based treatment planning. Cordoba et al. reported stage IIIA adenocarcinoma of a case of uterus didelphys (class III Müllerian duct anomaly). A total of $20 \mathrm{~Gy}$ were delivered to the high- risk clinical target volume (HR-CTV [26]) with BT, using the mold and pulsed-dose-rate techniques [23]. Ishibashi et al. reported the dose-volume histogram comparisons with tandem insertion into the right versus left uterine canals. The comparisons showed wide variations in the projected dose to the CTV and organ at risk. Tandem positioning was chosen to optimize the dose-volume distribution [25].

The standard treatment of locally advanced uterine cervical cancer is CCRT $[27,28]$. In our institution, 5-6 courses of CDDP $30 \mathrm{mg} / \mathrm{m}^{2}$ has been used mainly for CCRT [29]. Retrospective studies have also reported the use of CDDP $30 \mathrm{mg} / \mathrm{m}^{2}$ [30,31]. In Japan, the standard radiotherapy schedule for stage IIB (large tumor) is as follows: EBRT is given to the whole pelvis; a central shield is used after 30-40 Gy and at the same time, ICBT is commenced. The BT dose for point A corresponding to each central shielding doses are $24 \mathrm{~Gy} / 4-18 \mathrm{~Gy} / 3$ fractions [27]. The total doses for point A corresponding to these prescription doses are $62-64 \mathrm{~Gy}_{\mathrm{EQD} 2}$. Furthermore, when rectal or bladder doses are regarded as being equivalent to point $\mathrm{A}$, they are 72.4-73.2 $\mathrm{Gy}_{\mathrm{EQD} 2}$. Since the present case involved double cancers of uterus didelphys, standard therapy could not be applied; therefore, BT dose was increased once more than the standard therapy.

There is no standard RT for carcinoma of the cervix in a uterus didelphys, and the RT for carcinoma of the cervix in a uterus didelphys cannot simply be compared with the treatment for uterus simplex. In the present case, the total doses for point $A$, the rectum by the ICRU 38, the rectum by calculation and by dosimeter measurement, and the bladder by the ICRU 38 were 72.7, 107.9, 74.1, 64.0, and 107.6 $\mathrm{Gy}_{\mathrm{EQD} 2}$, respectively. Total doses for point $\mathrm{A}$, the rectum and bladder by ICRU 38 were high except for the rectum by dosimeter measurement, compared with the standard dose in Japan [27]. However, the dose was comparable to the values reported in literature, except for low-dose-rate cases $[20,22,24,25]$. Since the rectal doses by the ICRU 38 and by calculation were relatively high, the grade1 rectal late effect may have developed.

In our case, we discussed the treatment method. Two tandem applicators could not be inserted simultaneously in two uterine cavities with vaginal septum at ICBT, because of the anatomical position and the narrow cervical canal of each small uterus. Therefore, we used one tandem and two ovoid applicators in various ICBT combinations for four times. Only point A was used on the one side of the uterus as the reference point for all 4 sessions of BT; the left side of the uterus was used for three sessions, while the right side of the uterus was used for one session. Tandem insertion was performed three times in the large tumor of the left uterus, the ovoid applicators were put in the left vagina during the first insertion, and bilateral vagina in the second to third insertions, but the dose distribution was focused on the left uterus. In the last (fourth) insertion, a tandem was inserted to the right side to improve the dose distribution in the right uterine body to the uterine fundus. The two uteri were small, and their sounding lengths were $3.5 \mathrm{~cm}$ at BT. In Figure 4, which shows the uteri with reference to MRI immediately 
after the second ICBT, the dose at the right uterine fundus is somewhat lower compared with the left side. However, the tumor contour created with reference to MRI immediately after the second ICBT was surrounded by the distribution of $6 \mathrm{~Gy}$ in each of the four ICBT. Thus, the dose distribution is considered to be good for the tumor control on uterus didelphys, because there was no recurrence, even though grade 1 radiation proctitis occurred. The fact that the two uteri were small would likely be one of the reasons why the uterine body to the uterine fundus received a sufficient dose distribution.

At that time, only 2D treatment planning was used in our hospital. In Japan, a nationwide survey was performed in 2012, and 84\% (113/135) of BT treatment institutions reported that they used X-ray films for treatment planning [32]. On the other hand, a recent national survey of intracavitary BT in Japan in 2016 showed that $40 \%$ $(46 / 115)$ of BT treatment institutions used X-ray films for treatment planning and in $60 \%(69 / 115)$ of centers, CT images were acquired after applicator insertion. Three-dimensional planning was performed in $48 \%$ (55/115) [33].

In future, the use of IGBT would allow for the examination of the optimal dose distribution for the tumor and reduction of the dose to the rectum and bladder. That is, for such a special case, by examining the dose distribution for every intracavitary irradiation, it is possible to discuss how the applicator can be inserted at the next intracavitary irradiation to prescribe the optimal dose for HR-CTV.

\section{Conclusions}

The diagnosis and treatment of cancer of uterus didelphys with vaginal didelphys are very difficult. A careful medical interview and pelvic examination of the patient are required at the first visit. In the present case, a combination of EBRT and bilateral uterus BT using 2D treatment planning with concurrent chemotherapy was effective, with no evidence of recurrence or major late complications of the bladder and rectum 80 months after the start of treatment.

\section{Disclosure}

Authors report no conflict of interest.

\section{References}

1. Loring TW. Pregnancy and uterine malformations. A report of two unusual cases. Am J Obstet Gynecol 1973; 116: 505-510.

2. Schweid AI, Smith MR, Figge DC. Symmetrical epithelial reactions in the cervices of didelphic uteri: a field response. Acta Cytol 1968; 12: 406-409.

3. Pecorelli S. Revised FIGO staging for carcinoma of the vulva, cervix, and endometrium. Int J Gynaecol Obstet 2009; 105: 103-104.

4. Jarcho J. Malformations of the uterus; review of the subject, including embryology, comparative anatomy, diagnosis and report of cases. Am J Surg 1946; 71: 106-166.

5. The American Fertility Society. The American Fertility Society classifications of adnexal adhesions, distal tubal occlusion, tubal occlusion secondary to tubal ligation, tubal pregnancies, Müllerian anomalies and intrauterine adhesions. Fertil Steril 1988; 49: 944-955.

6. Kaneyasu Y, Nagai N, Nagata Y et al. Intra-arterial infusion chemotherapy using cisplatin with radiotherapy for stage III squamous cell carcinoma of the cervix. Int J Radiat Oncol Biol Phys 2009; 75: 369-377.

7. Quinn MA, Benedet JL, Odicino F et al. Carcinoma of the cervix uteri. FIGO26th Annual Report on the Results of Treatment in Gynecological Cancer. Int J Gynecol Obstet 2006; 95 Suppl 1: S43-S103.

8. ICRU Reports 38. Dose and volume specification for reporting intracavitary therapy in gynecology, 1985.

9. Cox JD, Stetz J, Pajak TF. Toxicity criteria of the radiation therapy oncology group (RTOG) and the European organization for research and treatment of cancer (EORTC). Int J Rad Oncol Biol Phys 1996; 31: 1341-1346.

10. Fox S, Mones JM, Kronstadt R et al. Bilateral and synchronous squamous cell carcinoma of the cervix in a patient with uterus didelphys. Obstet Gynecol 1986; 67: 76S-79S.

11. Bakri Y, Salem H, Said et al. Bilateral and synchronous cervical carcinoma in situ in a didelphic uterus. Int J Gynecol Obstet 1992; 37: 289-291.

12. Sugimori $H$, Hachisuga $T$, Nakamura $S$ et al. Cervical cancers in uterus didelphys. Gynecol Oncol 1990; 36: 439-443.

13. Woods MS, Sheppard RG, Hardman DA, Woods HJ. Congenital genitourinary anomalies. Is there a predilection for multiple primary malignant neoplasms? Cancer 1992; 69: 546549.

14. Corbett PJ, Crompton AC. Invasive carcinoma of one cervix in a uterus didelphys. Case report. Br J Obstet Gynaecol 1982; 89: 171-172.

15. David MP, Persitz E. Adenocarcinoma in a uterus duplex. Int Surg 1974; 59: 430-431.

16. Davies ST, Nisbet JD. Uterus didelphys with adenoacanthoma involving one cavity. Am J Obstet Gynecol 1972; 112: 1134-1136.

17. Braun RD. Uterus didelphys and endometrial carcinoma. A case report. Obstet Gynecol 1970; 35: 93-95.

18. Anneberg AD. Double vagina with double uterus (didelphys) containing endometrial adenocarcinoma. Report of a case. J Iowa Med Soc 1971; 61: 674-675.

19. Gauwerky F. Double cervical carcinoma in a case of double uterus and subseptate vagina. Zentralbl Gynakol 1955; 25: 967-971.

20. Lee CD, Churn M, Haddad N et al. Case report: Bilateral radical radiotherapy in a patient with uterus didelphys. $\mathrm{Br}$ J Radiol 2000; 73: 553-556.

21. Loo HW, Locks SM. Squamous cell carcinoma of the cervix: report of an unusual case of bicornuate bicollis uterus treated with bilateral intracavity brachytherapy. Br J Radiol 2010; 83: e143-e146.

22. Platta CS, Wallace C, Gondi V et al. Cervical brachytherapy technique for locally advanced carcinoma of the cervix in a patient with septate uterus. J Contemp Brachytherapy 2014; 6: 76-81.

23. Cordoba A, Escande A, Comte P et al. Locally advanced adenocarcinoma of the cervix on uterus didelphys: a case report. J Contemp Brachytherapy 2017; 9: 71-76.

24. Yavas G, Yavas C, Inan G. Locally advanced squamous cell cervical cancer in a patient with septate uterus. J Contemp Brachytherapy 2017; 9: 487-489.

25. Ishibashi N, Maebayashi T, Aizawa T et al. Brachytherapy for cervical cancer in septate uterus: Dose-volume differences with tandem implant placement in right vs. left uterine canal: A case report. Oncol Lett 2018; 15: 7273-7278.

26. Pötter R, Haie-Meder C, Van Limbergen E et al. Recommendations from gynaecological (GYN) GEC ESTRO working group (II): Concepts and terms in 3D image-based treatment planning in cervix cancer brachytherapy-3D dose volume parameters and aspects of 3D image-based anatomy, radiation physics, radiobiology. Radiother Oncol 2006; 78: 67-77. 
27. Ebina Y, Mikami M, Nagase S et al. Japan Society of Gynecologic Oncology guidelines 2017 for the treatment of uterine cervical cancer. Int J Clin Oncol 2019; 24: 1-19.

28. Vale C, Tierney JF, Stewart LA et al. Chemoradiotherapy for cervical cancer meta-analysis collaboration: reducing uncertainties about the effects of chemoradiotherapy for cervical cancer: individual patients data meta-analysis. Cochrane Database Syst Rev 2010; 1: CD008285.

29. Kaneyasu Y, Fujiwara H, Hirata E et al. Retrospective study on the usefulness of simultaneous chemoradiotherapy (CCRT) for advanced cervical cancer. Japn J Gynecol Oncol 2011; 29: 167-177.

30. Watanabe $Y$, Nakai H, Shimaoka M et al. Feasibility of concurrent cisplatin use during primary and adjuvant chemoradiation therapy: A phase I study in Japanese patients with cancer of the uterine cervix. Int J Clin Oncol 2006; 11: 309-313.

31. Ikushima H, Osaki K, Furutani S et al. Chemoradiation therapy for cervical cancer: toxicity of concurrent weekly cisplatin. Rad Med 2006; 24: 115-121.

32. Ohno T, Toita T, Tsujino K et al. A questionnaire-based survey on 3D image-guided brachytherapy for cervical cancer in Japan: Advances and obstacles. J Radiat Res 2015; 56: 897-903.

33. Toita $\mathrm{T}$, Ohno $\mathrm{T}$, Ikushima $\mathrm{H}$ et al. National survey of intracavitary brachytherapy for intact uterine cervical cancer in Japan. J Radiat Res 2018; 59: 469-476. 